\title{
Long-Haul Coherent Transmission Using a Silicon Nitride Microresonator-Based Frequency Comb as WDM Source
}

\author{
Attila Fülöp, ${ }^{* 1}$, Mikael Mazur ${ }^{1}$, Tobias A. Eriksson ${ }^{1}$, Peter A. Andrekson ${ }^{1}$, Victor Torres-Company ${ }^{1}$, \\ Pei-Hsun Wang ${ }^{2}$, Yi Xuan ${ }^{2,3}$, Dan E. Leaird ${ }^{2}$, Minghao $\mathbf{Q i}^{2,3}$, and Andrew M. Weiner ${ }^{2,3}$ \\ ${ }^{1}$ Photonics Laboratory, Department of Microtechnology and Nanoscience, Chalmers University of Technology, SE-41296 Gothenburg, Sweden \\ ${ }^{2}$ School of Electrical and Computer Engineering and ${ }^{3}$ Birck Nanotechnology Center, Purdue University, West Lafayette, IN 47907-2035, USA \\ *attila.fulop@chalmers.se
}

\begin{abstract}
We demonstrated transmission of polarization-multiplexed quadrature phase-shift keying data over $6000 \mathrm{~km}$ using a low-noise silicon nitride microresonator frequency comb as light source. These results show the technology's suitability for long-haul fiber communications. OCIS codes: (060.1660) Coherent communications; (190.4390) Nonlinear optics, integrated optics.
\end{abstract}

\section{Introduction}

Optical frequency combs have great potential as light sources in wavelength division multiplexing (WDM) fiber transmission systems. An optical frequency comb can replace hundreds of individual lasers and maintain the channel spacing by controlling its repetition rate. In addition, the broadband coherence of frequency combs can be used to mitigate nonlinear impairments upon fiber propagation [1]. Ideally, the frequency comb should be monolithically integrated with other transceiver components. In this regard, microresonator frequency combs based on silicon nitride offer a promising CMOS-compatible solution for lighting future fiber-communication systems [2]. Recent experiments indicate that the performance of this platform, in terms of optical signal to noise ratio (OSNR) and linewidth, is suitable for coherent communication systems over a few hundred kilometers [3,4].

In this work we demonstrate for the first time that microresonator frequency combs fulfill the requirements for use as WDM light sources in long-haul $(>>100 \mathrm{~km})$ transmission links. Specifically, we encoded data using polarization-multiplexed quadrature-phase shift keying (PM-QPSK) at 12.5 Gbaud on seven neighboring channels and successfully transmitted over more than $6000 \mathrm{~km}$ in a recirculating fiber loop. An important difference with respect to previous demonstrations is that the comb dynamics is based on modal interaction in a microresonator exhibiting global normal dispersion [5]. Linear coupling between higher-order transverse modes in the cavity introduces a spectral window of net anomalous dispersion that allows for switching on the comb via modulation instability [5]. As a result, the first oscillating lines correspond to the nearest neighbor longitudinal modes and the comb approaches a low-noise state in a deterministic manner [5,6]. The comb features long-term stability, high OSNR and high output power per line, which are critical to realize propagation over thousands of kilometers. This is the first demonstration of a CMOS-compatible multi-wavelength light source with a performance suitable for longhaul coherent communications.

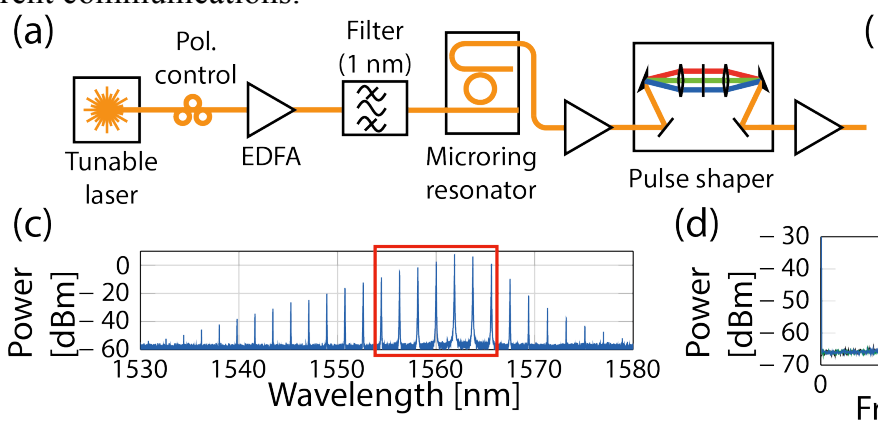

(b)

(d)
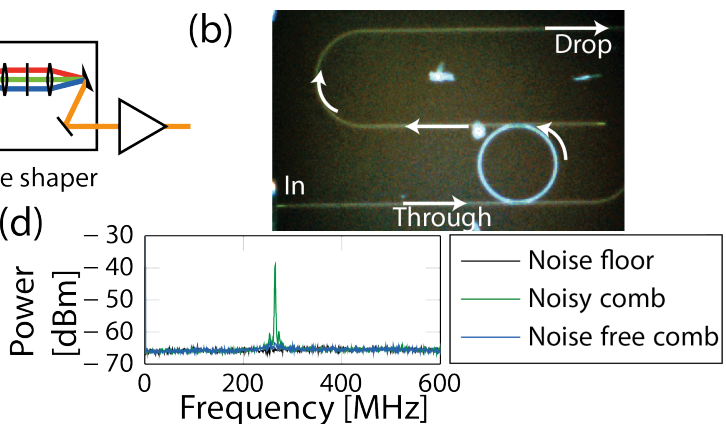

Fig. 1. (a) Sketch of the comb generation setup, including the flattening stage. The OSNR per line after the flattening was measured to be $37 \mathrm{~dB}$ at $0.1 \mathrm{~nm}$ resolution and the average power $9 \mathrm{dBm}$ per line. (b) Microscope image of the microring. (c) Initial optical spectrum of the comb with $0.01 \mathrm{~nm}$ resolution. The lines within the red box were used for transmission. (d) Radio-frequency spectrum with $1 \mathrm{MHz}$ RBW.

\section{Experiments}

The comb was generated, Fig. 1(a), by pumping a high-Q ( $>1$ million) silicon nitride microresonator (229 GHz free spectral range) with a single $100 \mathrm{kHz}$ linewidth continuous wave tunable laser amplified by an erbium-doped fiber amplifier (EDFA). Assuming $3 \mathrm{~dB}$ coupling loss, $24 \mathrm{dBm}$ is coupled into the bus waveguide. Using a filter before the resonator and extracting the comb from the resonator's drop port, see picture in Fig. 1(b), the amount of noise from the EDFA was minimized [7]. The laser approaches the resonance from the high-frequency side (blue detuned) 
while the noise is monitored with a radio-frequency (RF) spectrum analyzer. The optical spectrum of the resulting comb is shown in Fig. 1(c) and the RF spectrum in Fig. 1(d). To illustrate the need of careful pump laser tuning, Fig. 1(d) also contains an example of a noisy comb when the pump laser frequency was incorrect. The seven highest powered lines were then flattened using a pulse shaper.

(a)
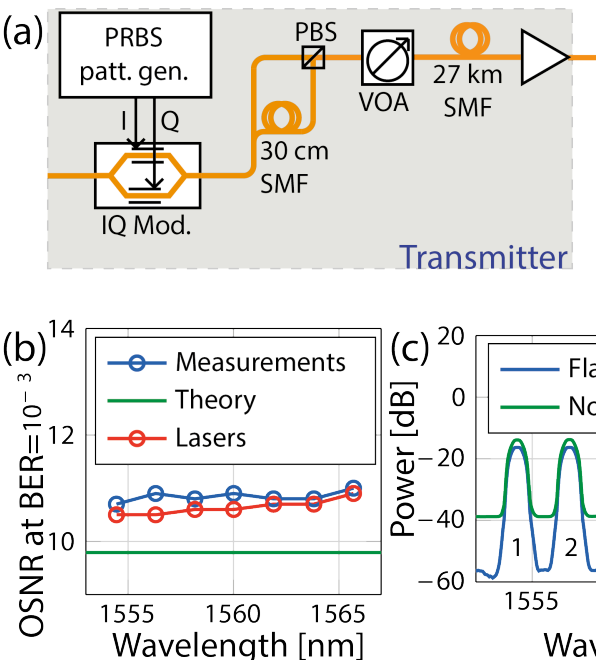

Transmitter

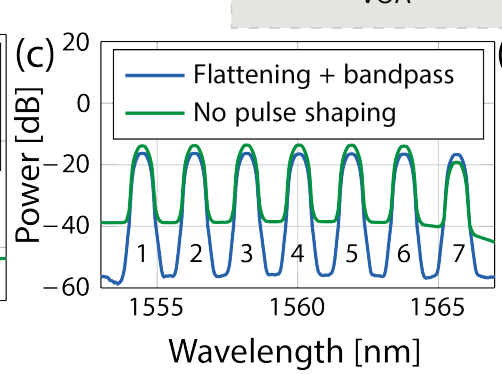

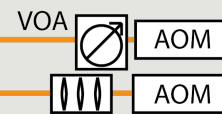

Pol. scrambler

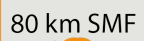

$80 \mathrm{~km} \mathrm{SM}$

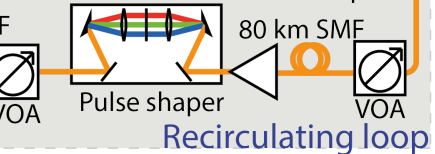

Recirculating loop

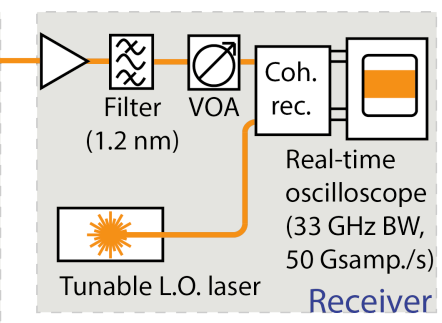

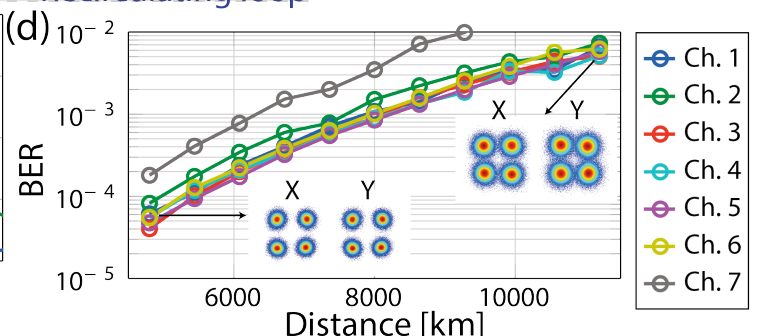

Fig. 2. (a) Sketch of the transmission setup. (b) Back-to-back noise-loading measurements comparing the comb to free-running lasers, showing an average implementation penalty of $1 \mathrm{~dB}$ compared to theory. (c) The optical spectrum after one roundtrip with and without pulse shaping at 0.5 $\mathrm{nm}$ resolution. (d) BER as a function of propagated distance. The insets show constellation diagrams for line 1 after $4800 \mathrm{~km}$ and $11200 \mathrm{~km}$.

The experimental transmission setup is shown in Fig. 2(a). 12.5 Gbaud QPSK data was modulated on all lines using a pseudorandom binary sequence (PRBS) source. A dual polarization signal was generated using a polarization multiplexing emulation stage with a delay longer than 10 symbols. The separate channels were then decorrelated utilizing the dispersion of a $27 \mathrm{~km}$ standard single-mode fiber (SMF). Figure 2(b) shows the results from a basic noise-loading measurement showing that the comb does not add significant penalty compared to free running lasers. The long-haul transmission itself was performed using a recirculating fiber loop. One roundtrip contained two spans of $80 \mathrm{~km} \mathrm{SMF}$, each of them preceded by a variable optical attenuator (VOA) for setting the launch powers, and a combination of an EDFA and a pulse shaper to ensure that the signal remained flat during the whole transmission. Apart from gain flattening, the pulse shapers also filtered out-of-band noise. Figure 2(c) shows the optical spectrum with and without pulse shaping after one roundtrip. The launched powers were optimized for a transmission distance of $8000 \mathrm{~km}$. The loop also contained a polarization scrambler synchronized to the roundtrip time to ensure that the polarization state into each roundtrip was different and acousto-optic modulators (AOMs) as loop switches. Finally, the receiver consisted of a tunable optical filter for measuring one channel at a time and a coherent receiver with a tunable $100 \mathrm{kHz}$ linewidth laser as local oscillator followed by a real-time oscilloscope. The sampled signal was then fed into an offline DSP algorithm containing IQ imbalance compensation, dispersion compensation, a constant-modulus-algorithm equalizer as well as a Viterbi-Viterbi-based phase tracker.

The calculated BER as a function of distance is shown in Fig. 2(d). It shows that the comb supports transmission over transatlantic distances for a received BER of $10^{-3}$, reaching a total data rate of $350 \mathrm{Gbit} / \mathrm{s}$. Channel 7, at 1565.7 $\mathrm{nm}$, suffered a penalty owing to it being located outside the specified gain bandwidth of the EDFAs, leading to a faster accumulation of noise, reaching $6000 \mathrm{~km}$ at the chosen BER, while the other channels reached up to $8000 \mathrm{~km}$. These results demonstrate, for the first time, the suitability of this integrated comb technology for long-haul coherent transmission.

\section{References}

[1] E. Temprana et al., "Overcoming Kerr-induced capacity limit in optical fiber transmission," Science 348, 1445 (2015).

[2] J. S. Levy et al., "CMOS-compatible multiple-wavelength oscillator for on-chip optical interconnects," Nature Photon. 4, 37 (2009).

[3] J. Pfeifle et al., "Coherent terabit communications with microresonator Kerr frequency combs," Nature Photon. 8, 375 (2014).

[4] J. Pfeifle at al., "Full C and L-band transmission at 20 Tbit/s using cavity-soliton Kerr ...," CLEO Postdeadline Papers, JTh5C.8 (2015).

[5] Y. Liu et al., "Investigation of mode coupling in normal-dispersion silicon nitride microresonators for Kerr ...," Optica 1, 137 (2014).

[6] P.-H. Wang et al., "Observation of correlation between route to formation, coherence, noise, and ...," Opt. Express 20, 29284 (2012).

[7] P.-H. Wang et al., "Drop-port study of microresonator frequency combs: power transfer, spectra and time-...,” Opt. Express 21, 22441 (2013).

We acknowledge support from the European Research Council (ERC-2011-AdG-291618 PSOPA), the Swedish Research Council (VR) and the KA Wallenberg foundation. The effort at Purdue was supported in part under grants from NSF (ECCS-150957), DARPA (W31P40-13-1-001.8) and AFOSR (FA9550-15-1-0211). 